\title{
Reining in uncontrolled inflammasome with PKA
}

\author{
Karen V Swanson \& Jenny P-Y Ting \\ New findings show that the NLRP3 inflammasome is inactivated by disassembly of the inflammasome mediated by the \\ kinase PKA and that this regulation might be negated in NLRP3-gain-of-function diseases.
}

Dimant uring the course of infection, the detection of pathogen-associated molecular patterns (PAMPs) or damage-associated molecular patterns (DAMPs) by patternrecognition receptors (PRRs) can lead to inflammasome formation, with subsequent activation of ccaspase-1, secretion of interleukin $1 \beta$ (IL-1 $\beta$ ) and IL-18, and cell death via pyroptosis ${ }^{1,2}$. However, this process must be tightly controlled, as uncontrolled inflammasome activation can lead to a plethora of disease outcomes. In this issue, Mortimer et al. describe how phosphorylation of the PRR NLRP3 by the kinase PKA, after inflammasome activation, attenuates the inflammasome to halt the inflammatory response ${ }^{3}$. Gain-of-function mutations in the gene encoding NLRP3 cause a family of related autoimmune disorders called 'CAPS' ('cryopyrin-associated periodic syndrome') that result in aberrant activation of the NLRP3 inflammasome with uncontrolled secretion of IL- $1 \beta$ and IL-18. The authors also provide insight into why some of the NLRP3 mutations that induce CAPS drive unregulated activation of the inflammasome ${ }^{3}$.

During pathogenic infection or sterile tissue damage, innate immune cells rely on PRRs located on or within them for detection of the insult to mount a proper immune response ${ }^{4}$. After sensing a PAMP or DAMP, subsets of each of the intracellular PRR families can induce the formation and activation of inflammasomes. The canonical inflammasome is a multimolecular protein structure composed of a PRR

Karen V. Swanson is with the Lineberger Comprehensive Cancer Center, University of North Carolina at Chapel Hill, Chapel Hill, North Carolina, USA, and the Department of Genetics, University of North Carolina at Chapel Hill, Chapel Hill, North Carolina, USA. Jenny P.-Y. Ting is with the Lineberger Comprehensive Cancer Center, University of North Carolina at Chapel Hill, Chapel Hill, North Carolina, USA, the Department of Genetics, University of North Carolina at Chapel Hill, Chapel Hill, North Carolina, USA, the Center for Translational Immunology, University of North Carolina at Chapel Hill, Chapel Hill, North Carolina, USA, and the Institute for Inflammatory Diseases, University of North Carolina at Chapel Hill, North Carolina, USA.

e-mail: jenny_ting@med.unc.edu sensor, usually the adaptor ASC ('apoptosisassociated speck-like protein containing a caspase-recruitment domain') and pro-caspase-1, which leads to the autocatalytic cleavage and activation of caspase-1 (Fig. 1). A noncanonical inflammasome is formed by intracellular sensing of lipopolysaccharide, which leads to activation of caspase-1 and caspase-11 (ref. 5). Inflammasomes induce the cleavage, activation and secretion of IL- $1 \beta$ and IL- 18 by caspase- 1 and also induce pyroptotic cell death.

The activation and resolution of inflammasomes are tightly controlled ${ }^{6,7}$. The activation of inflammasomes is controlled initially at the level of transcription, which is typically referred as 'signal one' in a two-step process. DAMPs, PAMPs or cytokines activate the transcription factor NF- $\kappa \mathrm{B}$ to upregulate the expression of genes encoding components of the inflammasome, particularly IL- $1 \beta$ and NLRP3. The second signal is composed of a DAMP or PAMP and shows specificity for the inflammasome that is activated. A prime example of this is the AIM2 inflammasome, which recognizes and binds DNA. However, the ligand for NLRP3 has remained undefined. While most of what is known about the regulation of the inflammasome addresses ways in which the cell prevents premature or unintentional inflammasome activation, less is known about how cells attenuate the inflammasome after it has been activated.

The finding that pathways that induce cAMP inhibit the release of IL- 1 is a longstanding observation $^{8}$. This inhibition has been shown to be specific for the NLRP3 inflammasome and possibly occurs through direct binding of cAMP to NLRP3 (ref. 9). Both prostaglandin and dopamine, through binding to their respective receptors, can induce cAMP and prevent activation of the NLRP3 inflammasome ${ }^{10,11}$. The binding of dopamine to one of its receptors, DRD1, which is found on the surface of innate immune cells, induces the production of cAMP, which in turn induces the ubiquitination and autophagy-mediated degradation of NLRP3 and thus prevents its activation. While what has been shown before is that cAMP prevents the formation of inflammasomes, Mortimer et al. are insightful in probing deeper into the theme of regulation of the NLRP3 inflammasome by cAMP ${ }^{3}$. It is important and different from previous studies not only because it furthers understanding of regulation of the NLRP3 inflammasome but also because it shows for the first time that an active NLRP3 inflammasome can be extinguished quickly. While it is well known that prostaglandins promote inflammation, they are also linked to its resolution. This current study shows that binding of prostaglandin $\mathrm{E}_{2}$ to its receptor EP4 induces cAMP and activation of PKA in bone-marrow-derived macrophages ${ }^{3}$. The authors show that inhibitors of PKA prevent the negative regulation of inflammasomes and that purified PKA can phosphorylate purified NLRP3. By immunoblot analysis, the authors show clear evidence for the disassembly of ASC oligomers after intracellular cAMP concentrations are increased (Fig. 1). This disassembly is dependent on PKAdirected phosphorylation of Ser295 within the nucleotide-binding domain of NLRP3, which prevents its ATPase activity.

Published studies have shown that cAMP directly prevents inflammasome activation without the need for PKA activation ${ }^{10,11}$. This new study ${ }^{3}$ suggests that after some NLRP3 agonists activate inflammasomes, the activation of PKA by cAMP and its direct phosphorylation of NLRP3 is needed to extinguish already activated inflammasomes ${ }^{3}$. Although important pieces of the mechanism are elucidated, many more questions are raised. For example, how does phosphorylated NLRP3 induce ASC disassembly? Because the ATPase activity of NLRP3 is important for inflammasome assembly, does the PKA-induced loss of ATPase activity in NLRP3 cause inflammasome disassembly? This hypothesis would support a published study showing that binding of ATPase and ATP by NLRP3 are important for inflammasome assembly ${ }^{12}$. Given what is known about inhibition of the NLRP3 inflammasome by cAMP, it would be useful to know whether mechanisms induced by both cAMP and PKA work together to limit inflammasome activity by different mechanisms. For example, does cAMP induce the ubiquitination of NLRP3 and target it to autophagosomes even when PKA is activated and phosphorylates NLRP3? It is possible that under different circumstances, such as dopamine-induced signaling versus prostaglandin-induced signaling, outcomes differ in 


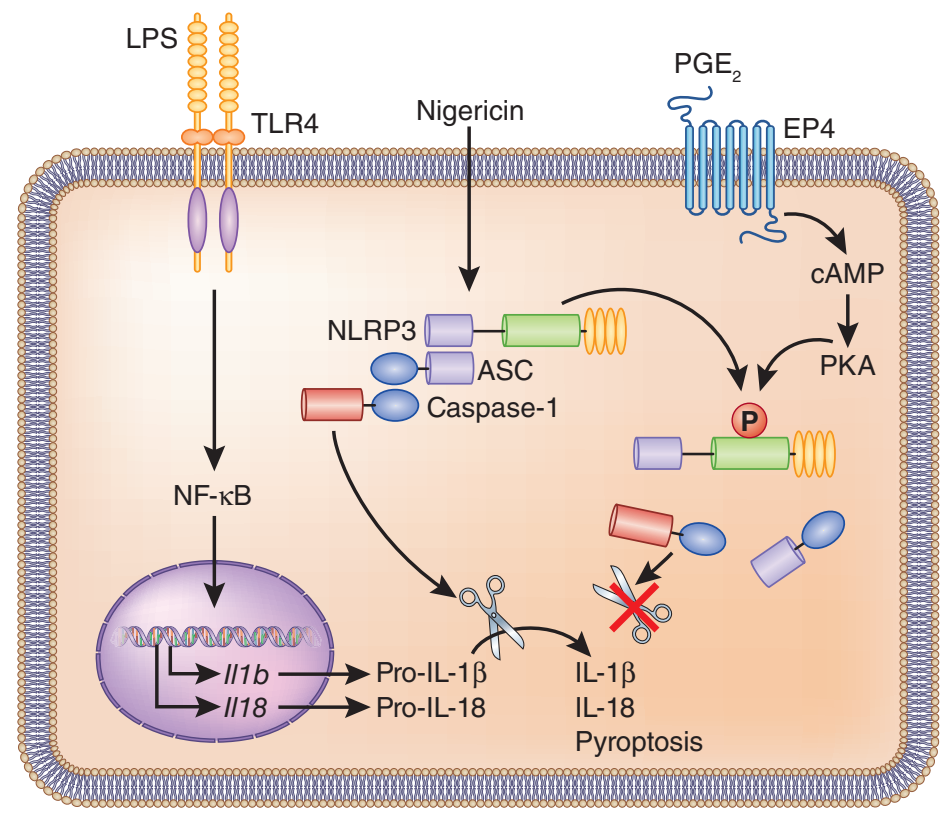

Figure 1 PKA negatively regulates the NLRP3 inflammasome. NLRP3 can sense a variety of PAMPs or DAMPs that induce assembly of the inflammasome. Activated caspase- 1 cleaves pro-IL-1 $\beta$ and pro-IL-18 to their active forms (IL-1 $\beta$ and IL-18, respectively), which induces their secretion. Caspase- 1 induces pyroptosis. Signaling via prostaglandin $\mathrm{E}_{2}\left(\mathrm{PGE}_{2}\right)$ induces cAMP formation and activates PKA, which directly phosphorylates NLRP3 in already formed inflammasomes. Phosphorylated NLRP3 induces disassembly of the inflammasome and loss of caspase-1 activity. LPS, lipopolysaccharide; TLR, Toll-like receptor.

that one or both mechanisms are activated and thus control the NLRP3 inflammasome differentially via the same tool: cAMP.

The study by Mortimer et al. also explores how the phosphorylation of NLRP3 by PKA might be important in autoinflammatory diseases mediated by gain of NLRP3 function ${ }^{3}$. Uncontrolled inflammation is linked to a multitude of diseases, while chronic inflammasome activation is known to contribute specifically to Alzheimer's disease, Parkinson's disease, type 2 diabetes, atherosclerosis and the CAPS family of autoimmune disorders ${ }^{1,13}$. The CAPS family of related disorders is caused mainly by gain-of-function single-nucleotide polymorphisms in NLRP3. The most serious of these is NOMID, which causes chronic production of IL- $1 \beta$ with potentially life-threatening outcomes, including severe arthritis, kidney damage, splenomegaly, chronic meningitis, uveitis, hearing loss, macrocephaly and other neurological problems. Mortimer et al. show that unlike wild-type NLRP3, which is phosphorylated on Ser295 by PKA to inactivate inflammasomes, a cluster of amino acid alterations near Ser295 encoded by CAPS-related single-nucleotide polymorphisms are resistant to negative regulation by $\mathrm{PKA}^{3}$. Thus, CAPS mutants might escape negative regulation and lose a molecular break, which results in disease manifestation.

This study highlights the importance of negative regulation of the NLRP3 inflammasome ${ }^{3}$. Previous work has indicated that CAPS arise as a result of gain-of-function single-nucleotide polymorphisms of NLRP3. However, this new work suggests that at least some of these single-nucleotide polymorphisms give rise to CAPS because the resulting NLRP3 mutants are unresponsive to negative regulation once activated $^{3}$. It will be interesting to see how many more of the more than 90 identified mutations that induce CAPS are due to lack of responsiveness to negative regulation. CAPS flare-ups can appear spontaneously or can be induced by known stressors such as cold, heat, fatigue or others. The new findings suggest that CAPS-related mutations might be more adept at escaping negative regulation of already activated inflammasomes and thus lead to inflammatory flares associated with the disorder.

\section{COMPETING FINANCIAL INTERESTS}

The authors declare no competing financial interests.

1. Guo, H., Callaway, J.B. \& Ting, J.P. Nat. Med. 21, 677-687 (2015)

2. Lamkanfi, M. \& Dixit, V.M. Cell 157, 1013-1022 (2014).

3. Mortimer, L., Moreau, F., MacDonald, J.A. \& Chadee, K. Nat. Immunol. 17, 1176-1186 (2106).

4. Takeuchi, O. \& Akira, S. Cell 140, 805-820 (2010).

5. Kayagaki, N. et al. Nature 479, 117-121 (2011).

6. Yang, J., Liu, Z. \& Xiao, T.S. Cell. Mol. Immunol. http:// dx.doi.org/10.1038/cmi.2016.29 (27 June 2016).

7. Latz, E., Xiao, T.S. \& Stutz, A. Nat. Rev. Immunol. 13 397-411 (2013).

8. Brandwein, S.R. J. Biol. Chem. 261, 8624-8632 (1986).

9. Lee, G.S. et al. Nature 492, 123-127 (2012).

10. Sokolowska, M. et al. J. Immunol. 194, 5472-5487 (2015).

11. Yan, Y. et al. Cell 160, 62-73 (2015).

12. Duncan, J.A. et al. Proc. Natl. Acad. Sci. USA 104 8041-8046 (2007).

13. Broderick, L., De Nardo, D., Franklin, B.S., Hoffman, H.M. \& Latz, E. Annu. Rev. Pathol. 10, 395-424 (2015). 